MATHEMATICS OF COMPUTATION

Volume 74, Number 250, Pages 655-679

S 0025-5718(04)01724-7

Article electronically published on November 2, 2004

\title{
NUMERICAL INDEFINITE INTEGRATION BY DOUBLE EXPONENTIAL SINC METHOD
}

\author{
KEN'ICHIRO TANAKA, MASAAKI SUGIHARA, AND KAZUO MUROTA
}

\begin{abstract}
We present a numerical method for approximating an indefinite integral by the double exponential sinc method. The approximation error of the proposed method with $N$ integrand function evaluations is

$$
\mathrm{O}\left(\exp \left(-c_{1} N / \log \left(c_{2} N\right)\right)\right)
$$

for a reasonably wide class of integrands, including those with endpoint singularities. The proposed method compares favorably with the existing formulas based on the ordinary sinc method. Computational results show the accordance of the actual convergence rates with the theoretical estimate.
\end{abstract}

\section{INTRODUCTION}

A variety of numerical methods based on sinc approximations has been studied during the last three decades $[6,7,8,9$. The methods cover function approximation, approximation of derivatives, approximate definite and indefinite integration, approximate solution of initial and boundary value ODE problems, and so on. In particular, the sinc interpolation formula is given by

$$
f(x) \approx \sum_{k=-N}^{N} f(k h) S(k, h)(x),
$$

where

$$
S(k, h)(x)=\frac{\sin [\pi(x-k h) / h]}{\pi(x-k h) / h} .
$$

The methods are collectively referred to as sinc numerical methods.

In this paper, we present a method and experimental results for approximating an indefinite integral $F(x)=\int_{-1}^{x} f(t) \mathrm{d} t$. In the literature [2, 3, 6], formulas for numerical indefinite integration based on the sinc approximation have been proposed, often based on a single exponential ("SE") transformation] such as $\psi_{1}(\zeta)=\tanh (\zeta / 2)$.

Received by the editor May 9, 2003.

2000 Mathematics Subject Classification. Primary 41A30, 41A25, 65D30.

Key words and phrases. Numerical indefinite integration, double exponential transformation, sinc numerical method.

This work is supported by the 21st Century COE Program on Information Science and Technology Strategic Core and a Grant-in-Aid of the Ministry of Education, Culture, Sports, Science and Technology of Japan.

${ }^{1}$ In contrast to the term "double exponential", we use "single exponential" when referring to the ordinary sinc method. 
To be specific, Stenger's formula [6] with this transformation is

$$
\begin{aligned}
& \int_{-1}^{x} f(t) \mathrm{d} t \\
= & \frac{\exp \left(A \psi_{1}^{-1}(x)\right)}{2 \cosh \left(A \psi_{1}^{-1}(x)\right)} h \sum_{k=-N}^{N} f\left(\psi_{1}(k h)\right) \psi_{1}^{\prime}(k h) \\
& +h \sum_{k=-N}^{N}\left[\sum _ { l = - N } ^ { N } \sigma _ { k - l } \left(f\left(\psi_{1}(l h)\right) \psi_{1}^{\prime}(l h)\right.\right. \\
& \left.\left.\quad-\frac{A}{2 \cosh ^{2}(A l h)} h \sum_{k=-N}^{N} f\left(\psi_{1}(k h)\right) \psi_{1}^{\prime}(k h)\right)\right] S(k, h)\left(\psi_{1}^{-1}(x)\right) \\
& +\mathrm{O}\left(\sqrt{N} \exp \left(-\sqrt{c_{f} N}\right)\right),
\end{aligned}
$$

where $\sigma_{k-l}$ is defined in (2.14) and $c_{f}$ is a constant that depends on the integrand $f$. The constant $A$ is determined appropriately according to the property of $f$. Such a formula has also been considered by Haber [2].

In place of the single exponential transformation, we employ in this paper a double exponential ("DE") transformation. Double exponential transformations are proposed by H. Takahasi and M. Mori 10, in designing a definite integration formula. Recently, it is known that the double exponential transformations are useful for various kinds of sinc numerical methods [4. We employ one of the double exponential transformations such as $\psi_{2}(\zeta)=\tanh ((\pi / 2) \sinh \zeta)$ to propose a more efficient formula:

$$
\begin{aligned}
& \int_{-1}^{x} f(t) \mathrm{d} t \\
= & \frac{1}{2}\left[\tanh \left(B \sinh \left(C \psi_{2}^{-1}(x)\right)\right)+1\right] h \sum_{k=-N}^{N} f\left(\psi_{2}(k h)\right) \psi_{2}^{\prime}(k h) \\
& +h \sum_{k=-N}^{N}\left[\sum _ { l = - N } ^ { N } \sigma _ { k - l } \left(f\left(\psi_{2}(l h)\right) \psi_{2}^{\prime}(l h)\right.\right. \\
& \left.\left.-\frac{B C \cosh (C l h)}{2 \cosh ^{2}(B \sinh (C l h))} h \sum_{k=-N}^{N} f\left(\psi_{2}(k h)\right) \psi_{2}^{\prime}(k h)\right)\right] S(k, h)\left(\psi_{2}^{-1}(x)\right) \\
& +\mathrm{O}\left(\exp \left[\frac{-c_{f}^{\prime} N}{\log \left(c_{f}^{\prime \prime} N\right)}\right]\right)
\end{aligned}
$$

where $c_{f}^{\prime}$ and $c_{f}^{\prime \prime}$ are constants that depend on the integrand $f$. The constants $B$ and $C$ are determined appropriately according to the property of $f$. The error term

$$
\mathrm{O}\left(\exp \left[\frac{-c_{f}^{\prime} N}{\log \left(c_{f}^{\prime \prime} N\right)}\right]\right)
$$

in this formula is smaller in order of magnitude than the error term

$$
\mathrm{O}\left(\sqrt{N} \exp \left(-\sqrt{c_{f} N}\right)\right)
$$


in the formula (1.3). Thus the proposed formula (1.4) is often more efficient than the formula (1.3), although there exist some functions (see Example 6 in Section 4) for which this is not the case.

The organization of this paper is as follows. In Section 2 we present our numerical indefinite integration formula on the entire real line $\mathbb{R}$ together with the main error estimate theorem. With the use of a double exponential transformation, the formula is adapted to the numerical indefinite integration formula on a finite interval in Section 3. In Section 4, we present some numerical results to confirm our theoretical error estimates. In Section 5 the proofs of the theorems and lemmas are provided. Concluding remarks are made in Section 6 .

\section{INDEFinite INTEGRATION ON THE ENTIRE REAL LINE}

In this section, we consider an indefinite integration on the entire real line $\mathbb{R}$ as the fundamental case of theoretical treatments. This case is essential in that the general case in Section 3 is a direct consequence of the results of this section in combination with the double exponential transformation.

Proofs of theorems are given in Section 5, unless otherwise indicated.

2.1. Notation. We introduce a function space as follows.

Definition 2.1. For a positive number $d$, a strip region $\mathcal{D}_{d}$ in $\mathbb{C}$ is defined as:

$$
\mathcal{D}_{d}=\{z \in \mathbb{C}|| \operatorname{Im} z \mid<d\} .
$$

Then, a function space $\mathbf{H}^{1}\left(\mathcal{D}_{d}\right)$ is defined as:

$$
\mathbf{H}^{1}\left(\mathcal{D}_{d}\right)=\left\{g \mid g \text { is analytic in } \mathcal{D}_{d}, N_{1}\left(g, \mathcal{D}_{d}\right)<\infty\right\},
$$

where

$$
\begin{aligned}
N_{1}\left(g, \mathcal{D}_{d}\right) & =\lim _{\varepsilon \rightarrow 0} \int_{\partial \mathcal{D}_{d}(\varepsilon)}|f(z)||\mathrm{d} z|, \\
\mathcal{D}_{d}(\varepsilon) & =\{z \in \mathbb{C}|| \operatorname{Re} z|<1 / \varepsilon,| \operatorname{Im} z \mid<d(1-\varepsilon)\} .
\end{aligned}
$$

In addition, we use operators $\mathcal{J}, C_{N, h}$, and $C_{h}$ for an indefinite integration and the sinc interpolation.

Definition 2.2. For a function $f$ defined on a complex region containing the real line $\mathbb{R}$, we define operators $\mathcal{J}, C_{N, h}$, and $C_{h}$ by

$$
\begin{aligned}
(\mathcal{J} f)(z) & =\int_{-\infty}^{z} f(t) \mathrm{d} t, \\
\left(C_{N, h} f\right)(z) & =\sum_{k=-N}^{N} f(k h) S(k, h)(z), \\
\left(C_{h} f\right)(z) & =\lim _{N \rightarrow \infty}\left(C_{N, h} f\right)(z),
\end{aligned}
$$

where $N$ is a positive integer and $h$ is a positive real number. 
2.2. Sinc interpolation. The sinc interpolation is a basic tool in the derivation of our formula. The interpolation error for a function of double exponential decay type is estimated as follows.

Theorem 2.1 ([9]). Assume that $f$ satisfies

$$
\begin{aligned}
& f \in \mathbf{H}^{1}\left(\mathcal{D}_{d}\right), \\
& \forall x \in \mathbb{R},|f(x)| \leq \alpha \exp (-\beta \exp (\gamma|x|))
\end{aligned}
$$

for some positive numbers $\alpha, \beta, \gamma$, and $d$. Then, there exists a positive number $c$, independent of $N$, such that

$$
\sup _{-\infty<x<\infty}\left|f(x)-\left(C_{N, h} f\right)(x)\right| \leq c \exp \left[\frac{-\pi d \gamma N}{\log (\pi d \gamma N / \beta)}\right],
$$

where

$$
h=\frac{\log (\pi d \gamma N / \beta)}{\gamma N} .
$$

2.3. Derivation of the indefinite integration formula on $\mathbb{R}$. We describe the derivation of the proposed formula on $\mathbb{R}$ and explain the basic idea behind it.

First, we apply the sinc interpolation to $(\mathcal{J} f)(x)=\int_{-\infty}^{x} f(t) \mathrm{d} t$ to obtain

$$
\begin{aligned}
(\mathcal{J} f)(x) & \approx\left(C_{N, h} \mathcal{J} f\right)(x) \\
& =\sum_{k=-N}^{N}(\mathcal{J} f)(k h) S(k, h)(x) \\
& =\sum_{k=-N}^{N}\left(\int_{-\infty}^{k h} f(t) \mathrm{d} t\right) S(k, h)(x) .
\end{aligned}
$$

Next, we apply the sinc interpolation to $f$ in the above expression to obtain

$$
\begin{aligned}
(\mathcal{J} f)(x) & \approx\left(C_{N, h} \mathcal{J} C_{N, h} f\right)(x) \\
& =\sum_{k=-N}^{N}\left[\int_{-\infty}^{k h} \sum_{l=-N}^{N} f(l h) S(l, h)(t) \mathrm{d} t\right] S(k, h)(x) \\
& =h \sum_{k=-N}^{N}\left[\sum_{l=-N}^{N} \sigma_{k-l} f(l h)\right] S(k, h)(x),
\end{aligned}
$$

where

$$
\sigma_{k-l}=\frac{1}{2}+\int_{0}^{k-l} \frac{\sin \pi t}{\pi t} \mathrm{~d} t .
$$

If $f$ and $\mathcal{J} f$ satisfy the assumptions of Theorem 2.1 then the approximations in (2.12) and (2.13), denoted " $\approx$ ", should work. We can apply Theorem 2.1 to obtain the following theorem. Its proof is omitted.

Theorem 2.2. Assume that $f$ and $\mathcal{J} f$ satisfy

$$
\begin{aligned}
& f \in \mathbf{H}^{1}\left(\mathcal{D}_{d}\right), \\
& \mathcal{J} f \in \mathbf{H}^{1}\left(\mathcal{D}_{d}\right), \\
& \forall x \in \mathbb{R},|f(x)| \leq \alpha \exp (-\beta \exp (\gamma|x|)), \\
& \forall x \in \mathbb{R},|(\mathcal{J} f)(x)| \leq \alpha \exp (-\beta \exp (\gamma|x|))
\end{aligned}
$$


for some positive numbers $\alpha, \beta, \gamma$, and $d$. Then, there exists a positive number $c$, independent of $N$, such that

$$
\sup _{-\infty<x<\infty}\left|(\mathcal{J} f)(x)-\left(C_{N, h} \mathcal{J} C_{N, h} f\right)(x)\right| \leq c \exp \left[\frac{-\pi d \gamma N}{\log (\pi d \gamma N / \beta)}\right]
$$

where

$$
h=\frac{\log (\pi d \gamma N / \beta)}{\gamma N} .
$$

The assumptions in the above theorem are stated in terms of both $f$ and $\mathcal{J} f$. It is, however, more natural to state the assumptions in terms of the given integrand $f$. We present the following theorem which imposes an additional condition $\lim _{x \rightarrow \infty}(\mathcal{J} f)(x)=0$. The general case, free from this additional condition, is presented in Theorem 2.4

Theorem 2.3. Assume that $f$ satisfies

$$
\begin{aligned}
& f \in \mathbf{H}^{1}\left(\mathcal{D}_{d}\right), \\
& \forall x \in \mathbb{R},|f(x)| \leq \alpha \exp (-\beta \exp (\gamma|x|)), \\
& \int_{-\infty}^{\infty} f(t) \mathrm{d} t=0
\end{aligned}
$$

for some positive numbers $\alpha, \beta, \gamma$, and $d$. Then, for any $\varepsilon$ with $0<\varepsilon<d$, there exists a positive number $c_{\varepsilon}$, independent of $N$, such that

$$
\sup _{-\infty<x<\infty}\left|(\mathcal{J} f)(x)-\left(C_{N, h} \mathcal{J} C_{N, h} f\right)(x)\right| \leq c_{\varepsilon} \exp \left[\frac{-\pi(d-\varepsilon) \gamma N}{\log (\pi(d-\varepsilon) \gamma N / \beta)}\right],
$$

where

$$
h=\frac{\log (\pi(d-\varepsilon) \gamma N / \beta)}{\gamma N} .
$$

We next treat the general case in which (2.23) is not assumed. We consider

$$
g(z)=f(z)-\kappa(z) \int_{-\infty}^{\infty} f(t) \mathrm{d} t
$$

with a function $\kappa$ such that $\int_{-\infty}^{\infty} \kappa(t) \mathrm{d} t=1$. Then we have

$$
\int_{-\infty}^{\infty} g(t) \mathrm{d} t=0
$$

To apply Theorem 2.3 to $g$ we must choose $\kappa$ so that (2.21) and (2.22) are satisfied for some $\alpha, \beta, \gamma$, and $d$. We consider

$$
\kappa(z)=\frac{B C \cosh (C z)}{2 \cosh ^{2}(B \sinh (C z))}\left(=\frac{\mathrm{d}}{\mathrm{d} z}\left[\frac{1}{2} \tanh (B \sinh (C z))+\frac{1}{2}\right]\right),
$$

parameterized by $B$ and $C$. We also introduce notation

$$
r(z)=\kappa(z) \int_{-\infty}^{\infty} f(t) \mathrm{d} t .
$$

The following proposition provides the decay rate of $\kappa$ and the function space that contains $\kappa$. The proof is straightforward and omitted. 
Proposition 2.1. Let $\beta_{\kappa}, \gamma_{\kappa}$, and $d_{\kappa}$ be determined as

$$
\begin{cases}\beta_{\kappa}=B-\varepsilon_{\beta}, \gamma_{\kappa}=C, d_{\kappa}=\frac{\pi}{2 C}-\varepsilon_{d}, & \text { if } 0<B<\frac{\pi}{2}, \\ \beta_{\kappa}=B-\varepsilon_{\beta}, \gamma_{\kappa}=C, d_{\kappa}=\frac{1}{C} \arcsin \left(\frac{\pi}{2 B}\right)-\varepsilon_{d}, & \text { if } \frac{\pi}{2} \leq B,\end{cases}
$$

where $\varepsilon_{\beta}$ and $\varepsilon_{d}$ are any positive numbers such that $\beta_{\kappa}>0$ and $d_{\kappa}>0$. Then we have

$$
\begin{aligned}
& \kappa \in \mathbf{H}^{1}\left(\mathcal{D}_{d_{\kappa}}\right) \\
& \forall x \in \mathbb{R},|\kappa(x)| \leq \alpha_{\kappa} \exp \left(-\beta_{\kappa} \exp \left(\gamma_{\kappa}|x|\right)\right) .
\end{aligned}
$$

To apply Theorem 2.3 to $g$, we need to determine the decay rate of $g$ and the function space to which $g$ belongs. The following lemma provides them. Its proof is easy and omitted.

Lemma 2.1. Let $\beta_{f}, \gamma_{f}$, and $d_{f}$ be constants such that

$$
\begin{aligned}
& f \in \mathbf{H}^{1}\left(\mathcal{D}_{d_{f}}\right), \\
& \forall x \in \mathbb{R},|f(x)| \leq \alpha_{f} \exp \left(-\beta_{f} \exp \left(\gamma_{f}|x|\right)\right),
\end{aligned}
$$

and let $\beta_{\kappa}, \gamma_{\kappa}$, and $d_{\kappa}$ be constants in (2.30). Then, for

$$
\begin{aligned}
\beta_{g} & = \begin{cases}\beta_{f}, & \text { if } \gamma_{f}<\gamma_{\kappa}, \\
\beta_{\kappa}, & \text { if } \gamma_{f}>\gamma_{\kappa}, \\
\min \left\{\beta_{f}, \beta_{\kappa}\right\}, & \text { if } \gamma_{f}=\gamma_{\kappa},\end{cases} \\
\gamma_{g} & =\min \left\{\gamma_{f}, \gamma_{\kappa}\right\}, \\
d_{g} & =\min \left\{d_{f}, d_{\kappa}\right\},
\end{aligned}
$$

we have

$$
\begin{aligned}
& g \in \mathbf{H}^{1}\left(\mathcal{D}_{d_{g}}\right), \\
& \forall x \in \mathbb{R},|g(x)| \leq \alpha_{g} \exp \left(-\beta_{g} \exp \left(\gamma_{g}|x|\right)\right) .
\end{aligned}
$$

Applying Theorem 2.3 to $g$, we immediately obtain the following theorem only with the assumptions (2.21) and (2.22) for $f$.

Theorem 2.4. Assume that $f$ satisfies

$$
\begin{aligned}
& f \in \mathbf{H}^{1}\left(\mathcal{D}_{d_{f}}\right), \\
& \forall x \in \mathbb{R},|f(x)| \leq \alpha_{f} \exp \left(-\beta_{f} \exp \left(\gamma_{f}|x|\right)\right)
\end{aligned}
$$


for some positive numbers $\alpha_{f}, \beta_{f}, \gamma_{f}$, and $d_{f}$. Then, for any $\varepsilon$ with $0<\varepsilon<d_{g}$ there exists a positive number $c_{\varepsilon}$, independent of $N$, such that

$$
\begin{aligned}
& \sup _{-\infty<x<\infty} \mid \int_{-\infty}^{x} f(t) \mathrm{d} t \\
& -\left[\frac{1}{2}[\tanh (B \sinh (C x))+1] \int_{-\infty}^{\infty} f(t) \mathrm{d} t\right. \\
& \quad+h \sum_{k=-N}^{N}\left[\sum_{l=-N}^{N} \sigma_{k-l}(f(l h)\right. \\
& \left.\left.\left.\quad-\frac{B C \cosh (C l h)}{2 \cosh ^{2}(B \sinh (C l h))} \int_{-\infty}^{\infty} f(t) \mathrm{d} t\right)\right] S(k, h)(x)\right] \mid \\
& =\sup _{-\infty<x<\infty}\left|(\mathcal{J} f)(x)-\left[(\mathcal{J} r)(x)+\left(C_{N, h} \mathcal{J} C_{N, h} g\right)(x)\right]\right| \\
& \leq c_{\varepsilon} \exp \left[\frac{-\pi\left(d_{g}-\varepsilon\right) \gamma_{g} N}{\log \left(\pi\left(d_{g}-\varepsilon\right) \gamma_{g} N / \beta_{g}\right)}\right]
\end{aligned}
$$

where

$$
h=\frac{\log \left(\pi\left(d_{g}-\varepsilon\right) \gamma_{g} N / \beta_{g}\right)}{\gamma_{g} N},
$$

and $\beta_{g}, \gamma_{g}$, and $d_{g}$ are taken as in (2.35) -(2.37).

In Theorem 2.4 the formula (2.42) contains the constant $\int_{-\infty}^{\infty} f(t) \mathrm{d} t$, which should be replaced by $h \sum_{k=-N}^{N} f(k h)$ in an actual computation.

Theorem 2.5. Under the same assumptions as in Theorem 2.4, the following estimate holds for some $c_{\varepsilon}^{\prime}$ :

$$
\begin{aligned}
& \sup _{-\infty<x<\infty} \mid \int_{-\infty}^{x} f(t) \mathrm{d} t \\
& -\left[\frac{1}{2}[\tanh (B \sinh (C x))+1] h \sum_{k=-N}^{N} f(k h)\right. \\
& +h \sum_{k=-N}^{N}\left[\sum_{l=-N}^{N} \sigma_{k-l}(f(l h)\right. \\
& \left.\left.\left.\quad-\frac{B C \cosh (C l h)}{2 \cosh ^{2}(B \sinh (C l h))} h \sum_{k=-N}^{N} f(k h)\right)\right] S(k, h)(x)\right] \mid \\
& \leq c_{\varepsilon}^{\prime} \exp \left[\frac{-\pi\left(d_{g}-\varepsilon\right) \gamma_{g} N}{\log \left(\pi\left(d_{g}-\varepsilon\right) \gamma_{g} N / \beta_{g}\right)}\right] .
\end{aligned}
$$

2.4. Optimal parameters. Given an integrand $f$, we are free to choose the parameters $B$ and $C$ in (2.30). We want to minimize the error (2.44) with respect to the parameters $B$ and $C$ for a given integrand $f$ satisfying (2.40) and (2.41). Hence, we are to determine the set of the parameter values $(B, C)$ that gives the maximum value of $\gamma_{g} d_{g}$, and then to choose a $(B, C)$, from among these maximizers, that makes $\beta_{g}$ as large as possible. Recall that $\beta_{g}, \gamma_{g}$, and $d_{g}$ are determined from $B$ and $C$ by Proposition 2.1 and Lemma 2.1. Here we note the following key fact. 
Proposition 2.2 ([8]). Assume that $f$ satisfies

$$
\begin{aligned}
& f \in \mathbf{H}^{1}\left(\mathcal{D}_{d_{f}}\right), \\
& \forall x \in \mathbb{R},|f(x)| \leq \alpha_{f} \exp \left(-\beta_{f} \exp \left(\gamma_{f}|x|\right)\right)
\end{aligned}
$$

for some positive numbers $\alpha_{f}, \beta_{f}, \gamma_{f}$, and $d_{f}$. If $f \not \equiv 0$, then $\gamma_{f} d_{f} \leq \pi / 2$.

Thus we may focus on the case $\gamma_{f} d_{f} \leq \pi / 2$. Then we determine $B$ and $C$ as follows. When $\gamma_{f} d_{f}<\pi / 2$,

$$
\begin{aligned}
& B=\frac{\pi}{2 \sin \left(\gamma_{f} d_{f}\right)}-\varepsilon_{B}, \\
& C=\gamma_{f}
\end{aligned}
$$

are the desired parameters, where $\varepsilon_{B}$ is any positive number such that $\pi / 2<B$.

Then we have

$$
\begin{aligned}
& \beta_{g}=\min \left\{\beta_{f}, \frac{\pi}{2 \sin \left(\gamma_{f} d_{f}\right)}-\varepsilon_{B}-\varepsilon_{\beta}\right\}, \\
& \gamma_{g}=\gamma_{f}, \\
& d_{g}=d_{f},
\end{aligned}
$$

where $\varepsilon_{\beta}$ is any positive number such that $\beta_{g}>0$.

When $\gamma_{f} d_{f}=\pi / 2$,

$$
\begin{aligned}
& B=\pi / 2, \\
& C=\gamma_{f}
\end{aligned}
$$

are the desired parameters. Then we have

$$
\begin{aligned}
& \beta_{g}=\min \left\{\beta_{f}, \frac{\pi}{2}-\varepsilon_{\beta}\right\}, \\
& \gamma_{g}=\gamma_{f} \\
& d_{g}=\frac{\pi}{2 \gamma_{f}}-\varepsilon_{d},
\end{aligned}
$$

where $\varepsilon_{\beta}$ and $\varepsilon_{d}$ are any positive numbers such that $\beta_{g}>0$ and $d_{g}>0$.

The explanation of the above argument is shown in subsection 5.3 .

2.5. Discussion. We discuss a technical difference between our setting and Stenger's 6]. Recall (2.40) and (2.41), our assumptions about the integrand $f$, in which the double exponential decay of $f$ is assumed only on the real line $\mathbb{R}$. On the other hand, the assumption in [6] is that

$$
f \in \mathbf{L}_{\alpha}\left(\mathcal{D}_{d}\right)
$$

for some positive numbers $\alpha$ and $d$, where $\mathbf{L}_{\alpha}\left(\mathcal{D}_{d}\right)$ is a function space of analytic functions $f$ on $\mathcal{D}_{d}$ satisfying

$$
\forall z \in \mathcal{D}_{d},|f(z)| \leq c \frac{\left|e^{\alpha z}\right|}{\left(1+\left|e^{z}\right|\right)^{2 \alpha}}
$$

for some positive number $c$. Thus the single exponential decay is assumed not only on the real line but also on the strip region $\mathcal{D}_{d}$. 
In parallel with $\mathbf{L}_{\alpha}\left(\mathcal{D}_{d}\right)$, we could have imposed the double exponential decay in a strip region and considered a function space $\mathbf{K}_{\beta, \gamma}\left(\mathcal{D}_{d}\right)$ of analytic functions $f$ on $\mathcal{D}_{d}$ satisfying

$$
\forall z \in \mathcal{D}_{d}, \quad|f(z)| \leq c \exp (-\beta \exp (\gamma|z|))
$$

for some positive number $c$. This function space, however, is less appropriate for the following reason. Consider $\kappa$ in (2.28) as a typical function with double exponential decay. We compare the tuple $\left(\beta_{\kappa}, \gamma_{\kappa}, d_{\kappa}\right)$ in (2.30) and a possible tuple $\left(\beta_{\kappa}^{\prime}, \gamma_{\kappa}^{\prime}, d_{\kappa}^{\prime}\right)$ such that $\mathbf{K}_{\beta_{\kappa}^{\prime}, \gamma_{\kappa}^{\prime}}\left(\mathcal{D}_{d_{\kappa}^{\prime}}\right)$ contains $\kappa$ in $(2.28)$. First, since $d_{\kappa}^{\prime}$ depends only on the strip region where $\kappa$ is analytic, we have

$$
d_{\kappa}^{\prime}= \begin{cases}\frac{\pi}{2 C}-\varepsilon_{d}, & \text { if } 0<B<\frac{\pi}{2}, \\ \frac{1}{C} \arcsin \left(\frac{\pi}{2 B}\right)-\varepsilon_{d}, & \text { if } \frac{\pi}{2} \leq B\end{cases}
$$

for some positive number $\varepsilon_{d}$. On the other hand, we can show

$$
\begin{aligned}
& \beta_{\kappa}^{\prime}<B \sin \left(\varepsilon_{d} C\right), \\
& \gamma_{\kappa}^{\prime}=C .
\end{aligned}
$$

Thus we cannot take $\beta_{\kappa}^{\prime}$ and $d_{\kappa}^{\prime}$ as large as $\beta_{\kappa}$ and $d_{\kappa}$ simultaneously. This implies that the alternative setting using $\mathbf{K}_{\beta_{\kappa}^{\prime}, \gamma_{\kappa}^{\prime}}\left(\mathcal{D}_{d_{\kappa}^{\prime}}\right)$ would lead to a poorer theoretical error bound than the present setting. In addition, the present setting, imposing a decay rate only on the real line, seems to be more natural, although this is certainly a matter of taste.

\section{IndeFinite INTEGRATION ON A FINITE INTERVAL}

Without loss of generality, we may use $[-1,1]$ as a finite interval. For the approximation of $F(x)=\int_{-1}^{x} f(t) \mathrm{d} t$, we take a double exponential transformation $z=\psi_{2}(\zeta)$, where

$$
\psi_{2}(\zeta)=\tanh \left[\frac{\pi}{2} \sinh \zeta\right]
$$

which maps $(-\infty, \infty)$ to $(-1,1)$. The following theorem gives an error estimate in the case of an indefinite integration on $[-1,1]$. Its proof is immediate from Theorem 2.5.

Theorem 3.1. Assume that, for a variable transformation $z=\psi_{2}(\zeta)$, the transformed function $\hat{f}(\zeta)=f\left(\psi_{2}(\zeta)\right) \psi_{2}^{\prime}(\zeta)$ satisfies

$$
\begin{aligned}
& \hat{f} \in \mathbf{H}^{1}\left(\mathcal{D}_{d_{\hat{f}}}\right), \\
& \forall x \in \mathbb{R},|\hat{f}(x)| \leq \alpha_{\hat{f}} \exp \left(-\beta_{\hat{f}} \exp \left(\gamma_{\hat{f}}|x|\right)\right)
\end{aligned}
$$


for some positive numbers $\alpha_{\hat{f}}, \beta_{\hat{f}}, \gamma_{\hat{f}}$, and $d_{\hat{f}}$. Then, for any $\varepsilon$ with $0<\varepsilon<d_{\hat{g}}$ there exists a positive number $c_{\varepsilon}$, independent of $N$, such that

$$
\begin{aligned}
& \sup _{-1<x<1} \mid \int_{-1}^{x} f(t) \mathrm{d} t \\
&- {\left[\frac{1}{2}\left[\tanh \left(B \sinh \left(C \psi_{2}^{-1}(x)\right)\right)+1\right] h \sum_{k=-N}^{N} f\left(\psi_{2}(k h)\right) \psi_{2}^{\prime}(k h)\right.} \\
&+h \sum_{k=-N}^{N}\left[\sum _ { l = - N } ^ { N } \sigma _ { k - l } \left(f\left(\psi_{2}(l h)\right) \psi_{2}^{\prime}(l h)\right.\right. \\
&\left.\left.\left.-\frac{B C \cosh (C l h)}{2 \cosh ^{2}(B \sinh (C l h))} h \sum_{k=-N}^{N} f\left(\psi_{2}(k h)\right) \psi_{2}^{\prime}(k h)\right)\right] S(k, h)\left(\psi_{2}^{-1}(x)\right)\right] \mid \\
& \leq c_{\varepsilon}^{\prime \prime} \exp \left[\frac{-\pi\left(d_{\hat{g}}-\varepsilon\right) \gamma_{\hat{g}} N}{\log \left(\pi\left(d_{\hat{g}}-\varepsilon\right) \gamma_{\hat{g}} N / \beta_{\hat{g}}\right)}\right]
\end{aligned}
$$

where

$$
\begin{aligned}
& \hat{g}=\hat{f}-r, \\
& h=\frac{\log \left(\pi\left(d_{\hat{g}}-\varepsilon\right) \gamma_{\hat{g}} N / \beta_{\hat{g}}\right)}{\gamma_{\hat{g}} N},
\end{aligned}
$$

and $B, C, \beta_{\hat{g}}, \gamma_{\hat{g}}$, and $d_{\hat{g}}$ are taken as in 2.47)-2.51), or 2.52 -2.56), with $f$ and $g$ replaced by $\hat{f}$ and $\hat{g}$, respectively.

The formula (3.4) has been presented in the Introduction as (1.4).

For the comparison between the "SE" formula (1.3) and the "DE" formula (1.4) in Section 4 we describe here the error estimate of (1.3) based on Stenger [6]. Recall the definition of $\mathbf{L}_{\alpha}\left(\mathcal{D}_{d}\right)$ in subsection 2.5.

Theorem 3.2. Assume that, for a variable transformation $z=\psi_{1}(\zeta)$, the transformed function $\breve{f}(\zeta)=f\left(\psi_{1}(\zeta)\right) \psi_{1}^{\prime}(\zeta)$ satisfies $\breve{f} \in \mathbf{L}_{\alpha_{\breve{f}}}\left(\mathcal{D}_{d_{\breve{f}}}\right)$ for some positive numbers $\alpha_{\breve{f}}$ and $d_{\breve{f}}$. Then, there exists a positive number $c$, independent of $N$, such that

$$
\begin{aligned}
& \sup _{-1<x<1} \mid \int_{-1}^{x} f(t) \mathrm{d} t \\
& -\left[\frac{\exp \left(A \psi_{1}^{-1}(x)\right)}{2 \cosh \left(A \psi_{1}^{-1}(x)\right)} h \sum_{k=-N}^{N} f\left(\psi_{1}(k h)\right) \psi_{1}^{\prime}(k h)\right. \\
& \quad+h \sum_{k=-N}^{N}\left[\sum _ { l = - N } ^ { N } \sigma _ { k - l } \left(f\left(\psi_{1}(l h)\right) \psi_{1}^{\prime}(l h)\right.\right. \\
& \left.\left.\left.\quad-\frac{A}{2 \cosh ^{2}(A l h)} h \sum_{k=-N}^{N} f\left(\psi_{1}(k h)\right) \psi_{1}^{\prime}(k h)\right)\right] S(k, h)\left(\psi_{1}^{-1}(x)\right)\right] \mid \\
& \leq c N^{1 / 2} \exp \left(-\sqrt{\pi \alpha_{\breve{f}}^{\prime} d_{\breve{f}}^{\prime} N}\right)
\end{aligned}
$$


where

$$
\alpha_{\breve{f}}^{\prime}=\min \left(\alpha_{\breve{f}}, 2 A\right), d_{\breve{f}}^{\prime}=\min \left(d_{\breve{f}}, \frac{\pi}{2 A}-\varepsilon_{d}\right), h=\sqrt{\frac{\pi d_{\breve{f}}^{\prime}}{\alpha_{\breve{f}}^{\prime} N}},
$$

and $\varepsilon_{d}$ is any positive number such that $d_{\breve{f}}^{\prime}>0$.

Since

$$
\alpha_{\breve{f}}^{\prime} d_{\breve{f}}^{\prime} \leq \min \left(\alpha_{\breve{f}} d_{\breve{f}}, \pi-2 A \varepsilon_{d}\right)
$$

by (3.8), the parameter $A$ given by

$$
A=\frac{\alpha_{\breve{f}}}{2}
$$

maximizes $\alpha_{\breve{f}}^{\prime} d_{\breve{f}}^{\prime}$, i.e., minimizes the error estimate in (3.7). Thus, setting $A$ as in (3.10), we obtain the best "SE" formula.

\section{Numerical Results}

In this section, we show numerical results of the formulas (1.3) and (1.4) to compare the actual errors of the two. We adopt the single exponential transformation

$$
\psi_{1}(\zeta)=\tanh (\zeta / 2)
$$

in the formula (1.3). The integrands used for numerical experiments are as follows:

Example 1.

$$
f_{1}(x)=\frac{1}{\pi \sqrt{1-x^{2}}}, \quad \int_{-1}^{x} f_{1}(t) \mathrm{d} t=\frac{1}{\pi}\left(\arcsin x+\frac{\pi}{2}\right),
$$

Example 2.

$$
\begin{aligned}
& f_{2}(x)= \frac{1}{4 \log 2} \log \left(\frac{1+x}{1-x}\right), \\
& \int_{-1}^{x} f_{2}(t) \mathrm{d} t=\frac{1}{4 \log 2}\left[\log (1+x)^{1+x}+\log (1-x)^{1-x}-2 \log 2\right],
\end{aligned}
$$

Example 3.

$$
f_{3}(x)=\frac{1}{2}, \quad \int_{-1}^{x} f_{3}(t) \mathrm{d} t=\frac{1}{2}(x+1),
$$

Example 4.

$$
f_{4}(x)=\frac{2}{\pi} \sqrt{1-x^{2}}, \quad \int_{-1}^{x} f_{4}(t) \mathrm{d} t=\frac{1}{\pi}\left(\arcsin x+x \sqrt{1-x^{2}}\right)+\frac{1}{2},
$$

Example 5.

$$
f_{5}(x)=\frac{2}{\pi\left(1+x^{2}\right)}, \quad \int_{-1}^{x} f_{5}(t) \mathrm{d} t=\frac{1}{2}+\frac{2}{\pi} \arctan x,
$$

Example 6.

$$
\begin{aligned}
f_{6}(x)= & -2\left[x \operatorname{cn}\left(2 \tanh ^{-1} x, \sqrt{0.5}\right)\right. \\
& \left.+\operatorname{dn}\left(2 \tanh ^{-1} x, \sqrt{0.5}\right) \operatorname{sn}\left(2 \tanh ^{-1} x, \sqrt{0.5}\right)\right], \\
\int_{-1}^{x} & f_{6}(t) \mathrm{d} t=\left(1-x^{2}\right) \operatorname{cn}\left(2 \tanh ^{-1} x, \sqrt{0.5}\right) .
\end{aligned}
$$


TABle 1. Parameter values $(\varepsilon=0.01)$

\begin{tabular}{|l|c|c|c|c|c|}
\hline & \multicolumn{2}{|c|}{ "SE" formula (1.3) } & \multicolumn{3}{|c|}{ "DE" formula (1.4) } \\
\cline { 2 - 6 } & $A$ & $h$ & $B$ & $C$ & $h$ \\
\hline Example 1 & $1 / 2$ & $\sqrt{\frac{2 \pi(\pi-\varepsilon)}{N}}$ & $\pi / 2-\varepsilon$ & 1 & $\frac{\log (2(\pi-4 \varepsilon) N)}{N}$ \\
Example 2 & 1 & $\sqrt{\frac{\pi(\pi-\varepsilon)}{(1-\varepsilon) N}}$ & $\pi / 2-\varepsilon$ & 1 & $\frac{\log [\pi(\pi / 2-2 \varepsilon) N /(\pi / 2-(\pi / 2) \varepsilon)]}{N}$ \\
Example 3 & 1 & $\sqrt{\frac{\pi(\pi-\varepsilon)}{N}}$ & $\pi / 2-\varepsilon$ & 1 & $\frac{\log ((\pi-4 \varepsilon) N)}{N}$ \\
Example 4 & $3 / 2$ & $\sqrt{\frac{2 \pi(2 \pi / 3-\varepsilon)}{3 N}}$ & $\pi / 2-\varepsilon$ & 1 & $\frac{\log ((\pi-2 \varepsilon) N)}{N}$ \\
Example 5 & 1 & $\sqrt{\frac{\pi(\pi / 2-\varepsilon)}{N}}$ & $\pi-\varepsilon$ & 1 & $\frac{\log ((\pi / 3-2 \varepsilon) N)}{N}$ \\
Example 6 & 1 & $\sqrt{\frac{\pi(K-\varepsilon)}{N}}$ & $\pi / 2-\varepsilon$ & 1 & $\frac{\log ((\pi / 2) N)}{N}$ \\
\hline
\end{tabular}

The functions $f_{1}, \ldots, f_{4}$ are taken from [2]. We take $N=1,4,9,16, \ldots, 100$ and take the necessary parameters according to Table 1 where

$$
K=\int_{0}^{\pi / 2} \frac{\mathrm{d} \theta}{\sqrt{1-0.5 \sin ^{2} \theta}}=1.85407 \cdots
$$

Let us explain how the parameter values for the "DE" formula (1.4) are determined with reference to the result of subsection 2.4. For $f_{1}$ in Example 1, we have

$$
\begin{aligned}
f_{1}(x) & =\mathrm{O}\left(\left|1-x^{2}\right|^{-1 / 2}\right)(x \rightarrow \pm 1) \\
& \Longrightarrow\left|f_{1}\left(\psi_{2}(x)\right) \psi_{2}^{\prime}(x)\right|=\mathrm{O}(\exp (-(\pi / 4) \exp |x|))(x \rightarrow \pm \infty \text { in } \mathbb{R}),
\end{aligned}
$$

which implies $\beta_{\hat{f}_{1}}=\pi / 4$ and $\gamma_{\hat{f}_{1}}=1$. Moreover, by (4.10) we have $d_{\hat{f}_{1}}=\pi / 2-\varepsilon_{d}$, where $0<\varepsilon_{d}<\pi / 2$. Then, by (2.47)-(2.51), we have

$$
B=\frac{\pi}{2 \sin \left(\pi / 2-\varepsilon_{d}\right)}-\varepsilon_{B}, C=1, \beta_{\hat{g}}=\frac{\pi}{4}, \gamma_{\hat{g}}=1, d_{\hat{g}}=\frac{\pi}{2}-\varepsilon_{d}
$$

where $\varepsilon_{B}$ is any positive number such that $B>0$. Since $B$ in (4.4) may be arbitrarily close to $\pi / 2$, we can set $B=\pi / 2-\varepsilon$ for any $\varepsilon$ with $0<\varepsilon<\pi / 2$. Finally, we have

$$
h=\frac{\log \left(\pi\left(d_{\hat{g}}-\varepsilon\right) \gamma_{\hat{g}} N / \beta_{\hat{g}}\right)}{\gamma_{\hat{g}} N}=\frac{\log (2(\pi-4 \varepsilon) N)}{N}
$$

when $\varepsilon_{d}=\varepsilon$.

The parameters for the other integrands are determined similarly. We note that Theorem 3.1 cannot be applied to the integrand $f_{6}$. More precisely, there exists no $d$ such that $\hat{f}_{6} \in \mathbf{H}^{1}\left(\mathcal{D}_{d}\right)$ for the transformed integrand $\hat{f}_{6}$ with double exponential decay. Thus the parameter values for $f_{6}$ have no theoretical justification. 
The parameter values for the "SE" formula (1.3) are based on Stenger [6]. To be more precise, for $f_{1}$ in Example 1, we have

$$
\left|f_{1}\left(\psi_{1}(z)\right) \psi_{1}^{\prime}(z)\right|=\mathrm{O}(\exp (-(1 / 2)|z|))\left(|z| \rightarrow \pm \infty \text { in } \mathcal{D}_{d_{\breve{f}_{1}}}\right)
$$

by (4.9), which implies that $\alpha_{\breve{f}_{1}}=1 / 2$. Moreover, by (4.9) we have $d_{\breve{f}_{1}}=\pi-\varepsilon_{d}$, where $0<\varepsilon_{d}<\pi$. Then, by (3.8) and (3.10) we have

$$
\alpha_{\breve{f}_{1}}^{\prime}=\frac{1}{2}, d_{\breve{f}_{1}}^{\prime}=\pi-\varepsilon_{d},
$$

and

$$
h=\sqrt{\frac{\pi d_{\breve{f}}^{\prime}}{\alpha_{\breve{f}}^{\prime} N}}=\sqrt{\frac{2 \pi(\pi-\varepsilon)}{N}},
$$

where $\varepsilon_{d}=\varepsilon$. The parameters for the other integrands are determined in a similar manner.

The values of the functions are evaluated at 379 points from $[-1,1]$ chosen as follows:

$$
x=0.00, \pm 0.01 k(k=1,2, \ldots, 90), \pm(1-0.001 k)(k=1,2, \ldots, 99) .
$$

The points are denser near the endpoints of the interval. We perform double precision floating-point computation. We compute the absolute errors at the above points and show the maximum values of them in Figures 1 6. "SE" and "DE" indicate the results of the examples by (1.3) and (1.4), respectively.

The integrands $f_{1}$ and $f_{2}$ tend to infinity near the endpoints of $[-1,1]$. To avoid cancellation of significant digits near $x= \pm 1$, we change the expressions of $f_{i}\left(\psi_{1}(z)\right) \psi_{1}^{\prime}(z)$ and $f_{i}\left(\psi_{2}(z)\right) \psi_{2}^{\prime}(z)$. For example,

$$
\begin{aligned}
f_{1}\left(\psi_{1}(z)\right) \psi_{1}^{\prime}(z) & =\frac{1}{\pi \sqrt{1-\tanh ^{2}(z / 2)}} \frac{1}{2 \cosh ^{2}(z / 2)} \\
& =\frac{1}{2 \pi \cosh (z / 2)}, \\
f_{1}\left(\psi_{2}(z)\right) \psi_{2}^{\prime}(z) & =\frac{1}{\pi \sqrt{1-\tanh ^{2}((\pi / 2) \sinh z)}} \frac{\pi \cosh z}{2 \cosh ^{2}((\pi / 2) \sinh z)} \\
& =\frac{\cosh z}{2 \cosh ((\pi / 2) \sinh z)} .
\end{aligned}
$$

The integrand $f_{2}$ tends to infinity more mildly than $f_{1}$, and the approximation of $f_{2}$ is better than the one of $f_{1}$. It is natural that the integrand $f_{3}$ is approximated very well because it has no singularities. Although the integrand $f_{4}$ has singularities at $x= \pm 1$, it is also approximated very well. It is well known that the double exponential formula is also effective for such an integrand.

From Examples 1-4, we can observe that the approximation errors hardly depend on the nonanalyticity of the integrands at the endpoints of the interval. 


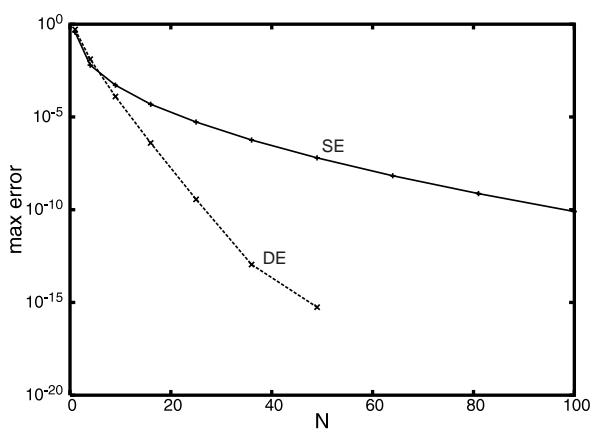

Figure 1. Example 1

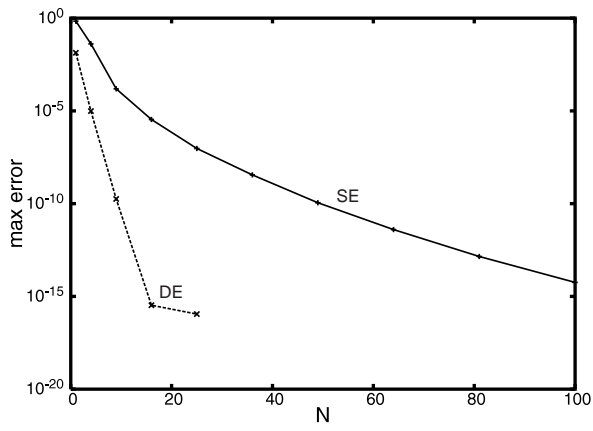

Figure 3. Example 3

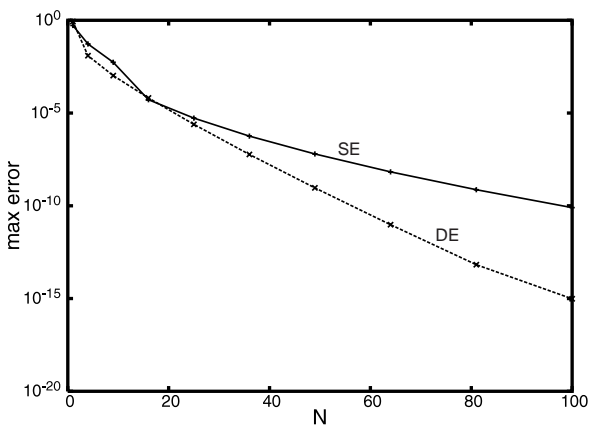

Figure 5. Example 5

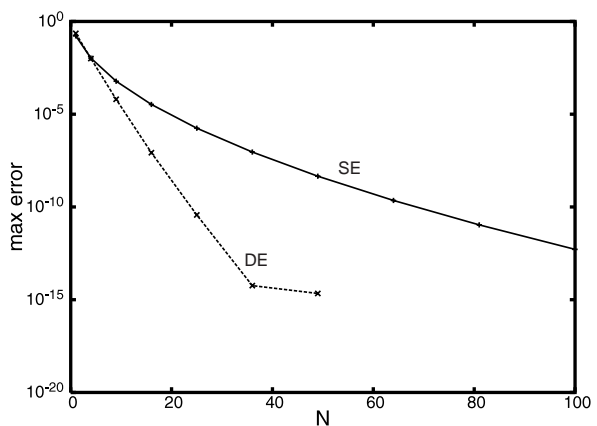

Figure 2. Example 2

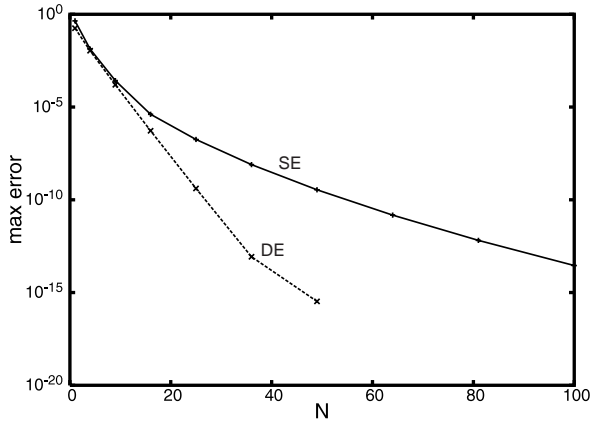

Figure 4. Example 4

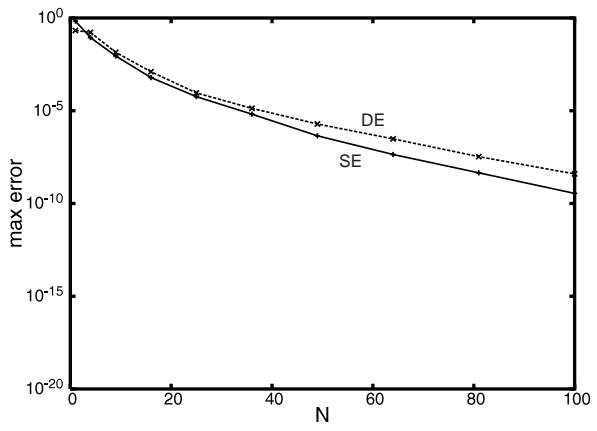

Figure 6. Example 6

Here, we note that $\gamma_{\hat{f}_{i}} d_{\hat{f}_{i}}$ is arbitrarily near $\pi / 2$ for $i=1, \ldots, 4$, whereas $\gamma_{\hat{f}_{5}} d_{\hat{f}_{5}}$ is properly less than $\pi / 2$ as a consequence of the singularities of $f_{5}$ at $z= \pm \mathrm{i}$. We can observe that the location of singularities in $\mathbb{C}$ affects the approximation error of the formula in $\mathbb{R}$.

From Examples 1-5, we can observe that our formula provides the results expected from the estimation (3.4) and is more accurate than the single exponential formula.

Finally, we emphasize that the estimate (11.4) is not applicable to $f_{6}$, but the estimate (1.3) is valid for $f_{6}$. 


\section{Proofs}

In this section, we prove the theorems stated in Sections 2 and 3 .

\subsection{Proof of Theorem 2.3,}

Lemma 5.1 ([6]). For the operator norm of $C_{N, h}$ defined as

$$
\left\|C_{N, h}\right\|_{\infty}=\sup _{\sup _{-\infty<x<\infty}|f(x)| \leq 1}\left|\sum_{k=-N}^{N} f(k h) S(k, h)(x)\right|,
$$

we have

$$
\left\|C_{N, h}\right\|_{\infty} \leq \sup _{-\infty<x<\infty} \sum_{k=-N}^{N}|S(k, h)(x)| \leq \frac{2}{\pi}\{3+\log N\} .
$$

Lemma $5.2([6])$. Let $f$ satisfy that $f \in \mathbf{H}^{1}\left(\mathcal{D}_{d}\right)$. Then,

$$
\left|(\mathcal{J} f)(x)-\left(\mathcal{J} C_{h} f\right)(x)\right| \leq \frac{h N_{1}\left(f, \mathcal{D}_{d}\right)}{4 d \sinh (\pi d / h)} .
$$

Lemma $5.3([6])$. For

$$
J(k, h)(x)=\int_{-\infty}^{x} S(k, h)(t) \mathrm{d} t
$$

we have

$$
|J(k, h)(x)| \leq 1.1 h .
$$

Let $\varepsilon$ be an arbitrary positive number and put $d^{\prime}=d-\varepsilon / 2$.

Lemma 5.4. Under the conditions (2.21) and (2.22), there exists a positive number $M\left(d^{\prime}\right)$, depending on $d^{\prime}$, such that

$$
\forall z \in \overline{\mathcal{D}}_{d^{\prime}},|f(z)| \leq M\left(d^{\prime}\right) .
$$

Proof. For a fixed $z \in \overline{\mathcal{D}}_{d^{\prime}}$, by Cauchy's integral formula we obtain the inequality

$$
|f(z)| \leq \frac{2}{\pi(d(1-\delta)-|\operatorname{Im} z|)} \int_{\partial \mathcal{D}_{d}(\delta)}|f(\zeta)||\mathrm{d} \zeta|,
$$

where $\delta$ is a sufficiently small positive number and $\mathcal{D}_{d}(\delta)$ is defined in (2.4). Then we have

$$
\begin{aligned}
|f(z)| & \leq \lim _{\delta \rightarrow 0} \frac{2}{\pi(d(1-\delta)-|\operatorname{Im} z|)} \int_{\partial \mathcal{D}_{d}(\delta)}|f(\zeta)||\mathrm{d} \zeta| \\
& =\frac{2}{\pi(d-|\operatorname{Im} z|)} N_{1}\left(f, \mathcal{D}_{d}\right) \\
& \leq \frac{4}{\pi\left(d-d^{\prime}\right)} N_{1}\left(f, \mathcal{D}_{d}\right),
\end{aligned}
$$

which proves (5.6). 
Lemma 5.5. Assume that $f$ is analytic in $\overline{\mathcal{D}}_{d^{\prime}}$. Moreover, assume (2.22) and (5.66), and set

$$
B\left(\gamma, d^{\prime}, y\right)=\left[\cos (\gamma|y|)-\cot \left(\gamma d^{\prime}\right) \sin (\gamma|y|)\right] \beta
$$

Then we have

$$
|f(x+y \mathrm{i})| \leq M^{\prime} \exp \left(-B\left(\gamma, d^{\prime}, y\right) \exp (\gamma|x|)\right)
$$

for all $y$ such that $|y| \leq d^{\prime}$.

Put $d^{\prime \prime}=d^{\prime}-\varepsilon / 2$.

Lemma 5.6. Assume that $f$ is analytic in $\overline{\mathcal{D}}_{d^{\prime}}$. Under the conditions (2.23) and (5.8), there exists a positive number $\alpha^{\prime}$ such that

$$
\begin{aligned}
& \mathcal{J} f \in \mathbf{H}^{1}\left(\mathcal{D}_{d^{\prime \prime}}\right), \\
& \forall x \in \mathbb{R},|(\mathcal{J} f)(x)| \leq \alpha^{\prime} \exp (-\beta \exp (\gamma|x|)) .
\end{aligned}
$$

For the proof of Lemmas 5.5 and 5.6 we need Propositions 5.1 and 5.2 below. Let $a$ be a real number such that $0<a<\pi / 2$ and define the fan-shaped domain $\mathcal{F}_{a}$ as

$$
\mathcal{F}_{a}=\left\{z \in \mathbb{C} \mid \frac{\pi}{2}-a<\arg z<\frac{\pi}{2}\right\} .
$$

Proposition 5.1 (Phragmén-Lindelöf [1]). Assume that $f$ is analytic in $\mathcal{F}_{a}$ and continuous in $\overline{\mathcal{F}}_{a}$. In addition, assume

$$
\begin{aligned}
& \forall z \in \overline{\mathcal{F}}_{a},|f(z)| \leq M, \\
& \exists c<0, \quad f\left(r \exp \left(\mathrm{i} \phi_{a}\right)\right)=\mathrm{O}(\exp (c r))(r \rightarrow \infty),
\end{aligned}
$$

where $\phi_{a}=\frac{\pi}{2}-a$. Then,

$$
\exists M^{\prime}>0, \forall z \in \mathcal{F}_{a},|f(z)| \leq M^{\prime} \exp \left(\frac{\cos (\arg z)}{\cos \phi_{a}} c|z|\right) .
$$

Proposition 5.2 (Montel [1]). Assume that $f$ is analytic and bounded in $\{z \in$ $\left.\mathbb{C} \mid \operatorname{Re} z>x_{0}, y_{0} \leq \operatorname{Im} z \leq y_{1}\right\}$. If

$$
\lim _{\substack{\operatorname{Re} z \rightarrow \infty \\ \operatorname{Im} z=y_{2}}} f(z)=c
$$

for a fixed $y_{2}$ such that $y_{0}<y_{2}<y_{1}$, then $f(z)$ converges to $c$ as $\operatorname{Re} z \rightarrow \infty$ uniformly with respect to $\operatorname{Im} z$ such that $y_{0}<\operatorname{Im} z<y_{1}$.

Proof of Lemma 5.5. We consider the case where $(x, y)$ is in the first quadrant of $\overline{\mathcal{D}}_{d^{\prime}}$. The proofs of the other cases are similar. 


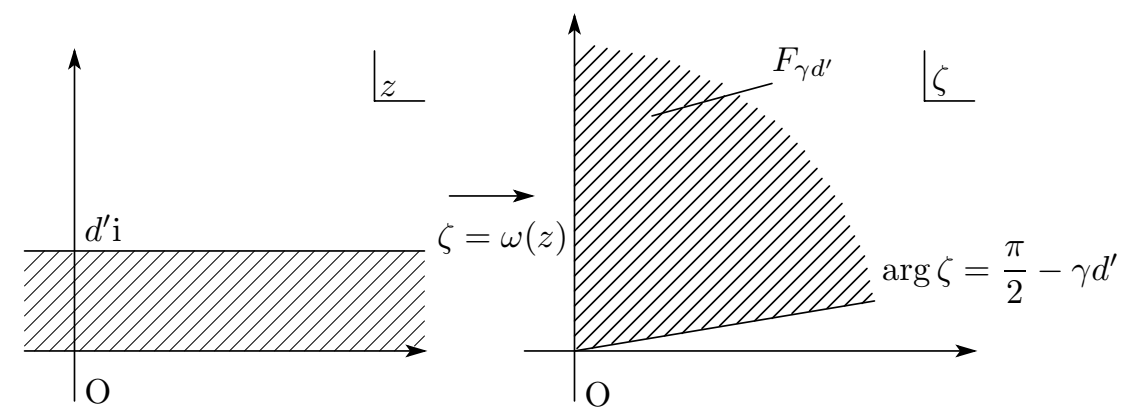

Figure 7 . Correspondence of the domains by $\omega$

We define the conformal mapping $\omega$ as

$$
\zeta=\omega(z)=\exp \left(\gamma z+\mathrm{i}\left(\frac{\pi}{2}-\gamma d^{\prime}\right)\right),
$$

and we set $\check{f}(\zeta)=f\left(\omega^{-1}(\zeta)\right)$. Let $z=x+y$ i and $\mathcal{F}_{\gamma d^{\prime}}$ be the domain shown in the right of Figure 7 We will apply Proposition 5.1 to $\check{f}$.

First, it is obvious that $\check{f}$ is analytic in $\mathcal{F}_{\gamma d^{\prime}}$ and continuous in $\overline{\mathcal{F}}_{\gamma d^{\prime}}-\{0\}$. Furthermore, the fact that $f(x)$ tends to zero as $x \rightarrow \pm \infty$, the boundedness of $f$ in $\overline{\mathcal{D}}_{d^{\prime}}$ (that is, (5.6)) and Proposition 5.2 guarantee the continuity of $\check{f}$ at the origin. Thus the analyticity of $\check{f}$ in $\mathcal{F}_{\gamma d^{\prime}}$ and the continuity of $\check{f}$ in $\overline{\mathcal{F}}_{\gamma d^{\prime}}$ are established.

Secondly, by (5.6) and the continuity of $\check{f}$ in $\overline{\mathcal{F}}_{\gamma d^{\prime}}$ we have

$$
\forall \zeta \in \overline{\mathcal{F}}_{\gamma d^{\prime}} \quad|\check{f}(\zeta)|=|f(z)| \leq M\left(d^{\prime}\right) .
$$

Lastly, it follows from

$$
|\zeta| \geq 1 \Rightarrow|\zeta|=\exp (\gamma x)=\exp (\gamma|x|)
$$

and (2.22) that

$$
\check{f}(\zeta)=\mathrm{O}(\exp (-\beta \exp (\gamma|x|)))=\mathrm{O}(\exp (-\beta|\zeta|)) \quad(|\zeta| \rightarrow \infty)
$$

holds for $\zeta$ with $\arg \zeta=\frac{\pi}{2}-\gamma d^{\prime}$.

Now all the assumptions of Proposition 5.1 for $\check{f}$ are established. Hence we have

$$
\forall \zeta \in \mathcal{F}_{\gamma d^{\prime}}, \quad|\check{f}(\zeta)| \leq M^{\prime} \exp \left(-\frac{\cos (\arg \zeta)}{\cos \left(\frac{\pi}{2}-\gamma d^{\prime}\right)} \beta|\zeta|\right) .
$$

Transforming this result to the $z$-plane, we finally have that

$$
\begin{aligned}
|f(z)| & \leq M^{\prime} \exp \left(-\frac{\cos \left(\frac{\pi}{2}-\gamma\left(d^{\prime}-y\right)\right)}{\cos \left(\frac{\pi}{2}-\gamma d^{\prime}\right)} \beta \exp (\gamma|x|)\right) \\
& =M^{\prime} \exp \left(-\frac{\sin \left(\gamma\left(d^{\prime}-y\right)\right)}{\sin \left(\gamma d^{\prime}\right)} \beta \exp (\gamma|x|)\right) \\
& =M^{\prime} \exp \left(-B\left(\gamma, d^{\prime}, y\right) \exp (\gamma|x|)\right)
\end{aligned}
$$

for all $z$ in $\overline{\mathcal{D}}_{d^{\prime}} \cap\{x+y \mathrm{i} \in \mathbb{C} \mid x \geq 0, y \geq 0\}$, which is the desired inequality. 


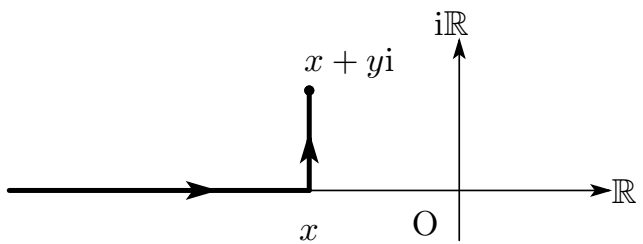

FIGURE 8. Integration path for computing $\int_{-\infty}^{z} f(\zeta) \mathrm{d} \zeta$

Proof of Lemma 5.6. Step 1. We estimate the value of

$$
\int_{-\infty}^{z} f(\zeta) \mathrm{d} \zeta
$$

when $z=x+y \mathrm{i} \in \mathcal{D}_{d^{\prime \prime}}$.

First, we consider the case where $x<0$ and $y \geq 0$. We have

$$
\begin{aligned}
\left|\int_{-\infty}^{z} f(\zeta) \mathrm{d} \zeta\right|= & \left|\int_{-\infty}^{x} f(s) \mathrm{d} s+\int_{0}^{y} f(x+t \mathrm{i}) \mathrm{id} t\right| \\
\leq & \int_{-\infty}^{x}|f(s)| \mathrm{d} s \\
& \quad+\int_{0}^{y}|f(x+t \mathrm{i})| \mathrm{d} t \\
\leq & M^{\prime}\left[\int_{-\infty}^{x} \exp (-\beta \exp (-\gamma s)) \mathrm{d} s\right. \\
& \left.\quad+\int_{0}^{y} \exp \left\{-\beta \frac{\exp (-\gamma x)}{\sin \left(\gamma d^{\prime}\right)} \sin \left(\gamma\left(d^{\prime}-t\right)\right)\right\} \mathrm{d} t\right]
\end{aligned}
$$

(see Figure 81). The first term of [ ] on the extreme right-hand side is estimated as follows:

$$
\begin{aligned}
\text { (first term) } & \leq \int_{-\infty}^{x} \exp (-\gamma s) \exp (-\beta \exp (-\gamma s)) \mathrm{d} s \\
& =\frac{1}{\beta \gamma} \int_{-\infty}^{x} \beta \gamma \exp (-\gamma s) \exp (-\beta \exp (-\gamma s)) \mathrm{d} s \\
& =\frac{1}{\beta \gamma} \exp (-\beta \exp (-\gamma x)) \\
& =\frac{1}{\beta \gamma} \exp (-\beta \exp (\gamma|x|)) .
\end{aligned}
$$

For the second term, we note that

$$
\begin{gathered}
\gamma \leq \frac{\pi}{2 d}, \quad 0 \leq t \leq d^{\prime \prime}<d^{\prime}<d \\
\Rightarrow \sin \left(\gamma\left(d^{\prime}-t\right)\right) \geq \frac{2}{\pi}\left(\gamma\left(d^{\prime}-t\right)\right),
\end{gathered}
$$


and obtain

$$
\begin{aligned}
\text { (second term) } & \leq \int_{0}^{y} \exp \left\{-\beta \frac{\exp (-\gamma x)}{\sin \left(\gamma d^{\prime}\right)} \frac{2}{\pi}\left(\gamma\left(d^{\prime}-t\right)\right)\right\} \mathrm{d} t \\
\leq & \int_{0}^{d^{\prime}-\varepsilon / 2} \exp \left\{-\beta \frac{\exp (-\gamma x)}{\sin \left(\gamma d^{\prime}\right)} \frac{2}{\pi}\left(\gamma\left(d^{\prime}-t\right)\right)\right\} \mathrm{d} t \\
= & \frac{\pi \sin \left(\gamma d^{\prime}\right)}{2 \beta \gamma} \exp (\gamma x) \\
& \cdot\left[\exp \left\{-\frac{\beta \gamma \varepsilon}{\pi \sin \left(\gamma d^{\prime}\right)} \exp (-\gamma x)\right\}-\exp \left\{-\frac{2 \beta \gamma d^{\prime}}{\pi \sin \left(\gamma d^{\prime}\right)} \exp (-\gamma x)\right\}\right] \\
= & \frac{\pi \sin \left(\gamma d^{\prime}\right)}{2 \beta \gamma} \exp (-\gamma|x|) \\
& \cdot\left[\exp \left\{-\frac{\beta \gamma \varepsilon}{\pi \sin \left(\gamma d^{\prime}\right)} \exp (\gamma|x|)\right\}-\exp \left\{-\frac{2 \beta \gamma d^{\prime}}{\pi \sin \left(\gamma d^{\prime}\right)} \exp (\gamma|x|)\right\}\right] .
\end{aligned}
$$

Next, we consider the case where $x \geq 0$ and $y \geq 0$. It follows from (2.23) that

$$
\begin{aligned}
\left|\int_{-\infty}^{z} f(\zeta) \mathrm{d} \zeta\right| & =\left|\int_{-\infty}^{x} f(s) \mathrm{d} s+\int_{0}^{y} f(x+t \mathrm{i}) \mathrm{id} t\right| \\
& =\left|-\int_{x}^{\infty} f(s) \mathrm{d} s+\int_{0}^{y} f(x+t \mathrm{i}) \mathrm{id} t\right| \\
& \leq \int_{x}^{\infty}|f(s)| \mathrm{d} s+\int_{0}^{y}|f(x+t \mathrm{i})| \mathrm{d} t .
\end{aligned}
$$

By applying (5.8) to the terms in the extreme right-hand side, we obtain the same bounds given in (5.16) and (5.17).

Lastly, we consider the case where $y<0$. Since the bound for

$$
\left|\int_{-\infty}^{z} f(\zeta) \mathrm{d} \zeta\right|
$$

should be symmetric with respect to the real axis, we have the same bounds given in (5.16) and (5.17).

Step 2. First, we prove (5.10). It follows from the results in the case where $y=0$ in Step 1 that $(\mathcal{J} f)(x)=\mathrm{O}(\exp (-\beta \exp (\gamma|x|)))$, i.e., (5.10).

Next, we prove (5.9). We take the contour shown in Figure 9 for computing

$$
N_{1}\left(\mathcal{J} f, \mathcal{D}_{d^{\prime \prime}}\right)
$$

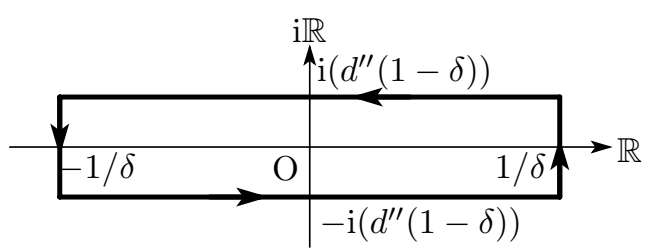

Figure 9. Contour for computing $N_{1}\left(\mathcal{J} f, \mathcal{D}_{d^{\prime \prime}}\right)$ 
where $\delta$ is a sufficiently small positive number. The integral considered here is

$$
\begin{aligned}
& \underbrace{\int_{-d^{\prime \prime}(1-\delta)}^{d^{\prime \prime}(1-\delta)}|(\mathcal{J} f)(-1 / \delta+y \mathrm{i})| \mathrm{d} y}_{I_{1}(\delta)}+\underbrace{\int_{-d^{\prime \prime}(1-\delta)}^{d^{\prime \prime}(1-\delta)}|(\mathcal{J} f)(1 / \delta+y \mathrm{i})| \mathrm{d} y}_{I_{2}(\delta)} \\
& +\underbrace{\int_{-1 / \delta}^{1 / \delta}\left|(\mathcal{J} f)\left(x-d^{\prime \prime}(1-\delta) \mathrm{i}\right)\right| \mathrm{d} x}_{I_{3}(\delta)}+\underbrace{\int_{-1 / \delta}^{1 / \delta}\left|(\mathcal{J} f)\left(x+d^{\prime \prime}(1-\delta) \mathrm{i}\right)\right| \mathrm{d} x}_{I_{4}(\delta)} .
\end{aligned}
$$

We set

$$
\begin{aligned}
& M_{1}(x)=(\text { the extreme right-hand side of }(\underline{5.160}), \\
& M_{2}(x)=(\text { the extreme right-hand side of }(\text { (5.17) }) .
\end{aligned}
$$

Note that $|(\mathcal{J} f)(z)| \leq M_{1}(x)+M_{2}(x)$ holds for all $z \in \mathcal{D}_{d^{\prime \prime}}$ as shown in Step 1. Then we have

$$
\begin{aligned}
& I_{1}(\delta)+I_{2}(\delta)+I_{3}(\delta)+I_{4}(\delta) \\
& \leq 4 d^{\prime \prime}\left(M_{1}(1 / \delta)+M_{2}(1 / \delta)\right)+2 \int_{x=-1 / \delta}^{x=1 / \delta}\left(M_{1}(x)+M_{2}(x)\right) \mathrm{d} x .
\end{aligned}
$$

Since

$$
\begin{aligned}
& M_{1}(x)=\mathrm{O}(\exp (-\beta \exp (\gamma|x|))), \\
& M_{2}(x)=\mathrm{o}(\exp (-\gamma|x|)),
\end{aligned}
$$

the right-hand side of (5.20) is bounded as $\delta$ approaches zero. Thus we have $\mathcal{J} f \in \mathbf{H}^{1}\left(\mathcal{D}_{d^{\prime \prime}}\right)$, i.e., (5.9) .

Proof of Theorem [2.3. Note that $d^{\prime \prime}=d-\varepsilon$. We have

$$
\begin{aligned}
& \left|(\mathcal{J} f)(x)-\left(C_{N, h} \mathcal{J} C_{N, h} f\right)(x)\right| \\
& \leq\left|(\mathcal{J} f)(x)-\left(C_{N, h} \mathcal{J} f\right)(x)\right|+\left|\left(C_{N, h} \mathcal{J} f\right)(x)-\left(C_{N, h} \mathcal{J} C_{h} f\right)(x)\right| \\
& \quad+\left|\left(C_{N, h} \mathcal{J} C_{h} f\right)(x)-\left(C_{N, h} \mathcal{J} C_{N, h} f\right)(x)\right| .
\end{aligned}
$$

We define $\varepsilon_{1}, \varepsilon_{2}$, and $\varepsilon_{3}$ as follows:

$$
\begin{aligned}
& \varepsilon_{1}(x)=(\mathcal{J} f)(x)-\left(C_{N, h} \mathcal{J} f\right)(x), \\
& \varepsilon_{2}(x)=\left(C_{N, h} \mathcal{J} f\right)(x)-\left(C_{N, h} \mathcal{J} C_{h} f\right)(x), \\
& \varepsilon_{3}(x)=\left(C_{N, h} \mathcal{J} C_{h} f\right)(x)-\left(C_{N, h} \mathcal{J} C_{N, h} f\right)(x) .
\end{aligned}
$$

To complete the proof, we estimate $\varepsilon_{1}, \varepsilon_{2}$, and $\varepsilon_{3}$ in turn.

Estimation of $\varepsilon_{1}$. By Lemma 5.6, we have

$$
\begin{aligned}
& \mathcal{J} f \in \mathbf{H}^{1}\left(\mathcal{D}_{d^{\prime \prime}}\right), \\
& \forall x \in \mathbb{R},|(\mathcal{J} f)(x)| \leq \alpha^{\prime} \exp (-\beta \exp (\gamma|x|)) .
\end{aligned}
$$

Therefore it follows from Theorem 2.1 that

$$
\left|\varepsilon_{1}(x)\right| \leq c_{1} \exp \left[\frac{-\pi d^{\prime \prime} \gamma N}{\log \left(\pi d^{\prime \prime} \gamma N / \beta\right)}\right] .
$$


Estimation of $\varepsilon_{2}$. By Lemma 5.2 we have

$$
\left|(\mathcal{J} f)(x)-\left(\mathcal{J} C_{h} f\right)(x)\right| \leq \frac{h N_{1}\left(f, \mathcal{D}_{d}\right)}{4 d \sinh (\pi d / h)} \leq c_{2} h \exp (-\pi d / h),
$$

where $c_{2}$ is a constant that is independent of $h$ (assuming, of course, that $h$ is uniformly bounded). With $h$ in (2.25), it follows from Lemma 5.1 that

$$
\begin{aligned}
\left|\varepsilon_{2}(x)\right| & =\left|\left(C_{N, h} \mathcal{J} f\right)(x)-\left(C_{N, h} \mathcal{J} C_{h} f\right)(x)\right| \\
& \leq\left\|C_{N, h}\right\|_{\infty} \sup _{-\infty<x<\infty}\left|(\mathcal{J} f)(x)-\left(\mathcal{J} C_{h} f\right)(x)\right| \\
& \leq \frac{2}{\pi}(3+\log N) \cdot c_{2} h \exp (-\pi d / h) \\
& =\frac{2}{\pi}(3+\log N) \cdot c_{2} \frac{\log \left(\pi d^{\prime \prime} \gamma N / \beta\right)}{\gamma N} \exp \left[\frac{-\pi d \gamma N}{\log \left(\pi d^{\prime \prime} \gamma N / \beta\right)}\right] \\
& \leq c_{3} \frac{(\log N)^{2}}{N} \exp \left[\frac{-\pi d \gamma N}{\log \left(\pi d^{\prime \prime} \gamma N / \beta\right)}\right],
\end{aligned}
$$

where $c_{3}$ is a constant that is independent of $N$.

Estimation of $\varepsilon_{3}$. By Lemma 5.3, we have

$$
\begin{aligned}
\mid\left(\mathcal{J} C_{h} f\right) & (x)-\left(\mathcal{J} C_{N, h} f\right)(x) \mid \\
& =\left|\int_{-\infty}^{x} \sum_{|k|>N} f(k h) S(k, h)(t) \mathrm{d} t\right|=\left|\sum_{|k|>N} f(k h) J(k, h)(x)\right| \\
& \leq \sup _{-\infty<x<\infty}|J(k, h)(x)| \sum_{|k|>N}|f(k h)| \leq 1.1 h \sum_{|k|>N} \alpha \exp (-\beta \exp (\gamma|k h|)) \\
& =2.2 \alpha h \sum_{k=N+1}^{\infty} \exp (-\beta \exp (\gamma k h)) \leq 2.2 \alpha h \int_{N}^{\infty} \exp (-\beta \exp (\gamma h t)) \mathrm{d} t \\
& \leq \frac{2.2 \alpha h}{\beta \gamma h \exp (\gamma h N)} \int_{N}^{\infty} \beta \gamma h \exp (\gamma h t) \exp (-\beta \exp (\gamma h t)) \mathrm{d} t \\
& =\frac{2.2 \alpha \exp (-\beta \exp (\gamma h N))}{\beta \gamma \exp (\gamma h N)} \\
& =\frac{2.2 \alpha}{\pi d^{\prime \prime} \gamma^{2}} \frac{1}{N} \exp \left(-\pi d^{\prime \prime} \gamma N\right)
\end{aligned}
$$

where $h$ is taken as (2.25). Then, analogously to (5.22), we have

$$
\begin{aligned}
\left|\varepsilon_{3}(x)\right| & =\left|\left(C_{N, h} \mathcal{J} C_{h} f\right)(x)-\left(C_{N, h} \mathcal{J} C_{N, h} f\right)(x)\right| \\
& \leq\left\|C_{N, h}\right\|_{\infty} \sup _{-\infty<x<\infty}\left|\left(\mathcal{J} C_{h} f\right)(x)-\left(\mathcal{J} C_{N, h} f\right)(x)\right| \\
& \leq c_{4} \frac{\log N}{N} \exp \left(-\pi d^{\prime \prime} \gamma N\right),
\end{aligned}
$$

where $c_{4}$ is a constant that is independent of $N$.

Combining (5.21), (5.22), and (5.23), we get (2.24). Thus the proof of Theorem $[2.3$ is completed. 
5.2. Proof of Theorem 2.5. We need the following proposition and lemma. The proof of the proposition is similar to that of Theorem 3.2 in [8] and hence is omitted.

Proposition 5.3. Assume that a function $f$ satisfies (2.40) and (2.41) for some $\alpha_{f}, \beta_{f}, \gamma_{f}$, and $d_{f}$. Then we have

$$
\left|\int_{-\infty}^{\infty} f(t) \mathrm{d} t-h \sum_{k=-N}^{N} f(k h)\right| \leq c \exp \left[\frac{-2 \pi d_{f} \gamma_{g} N}{\log \left(\pi\left(d_{g}-\varepsilon\right) \gamma_{g} N / \beta_{g}\right)}\right]
$$

for a constant $c$, independent of $N$, where we take $h$ as (2.43), i.e.,

$$
h=\frac{\log \left(\pi\left(d_{g}-\varepsilon\right) \gamma_{g} N / \beta_{g}\right)}{\gamma_{g} N} .
$$

Lemma 5.7. Let

$$
h=\frac{\log \left(\pi\left(d_{g}-\varepsilon\right) \gamma_{g} N / \beta_{g}\right)}{\gamma_{g} N} .
$$

Then

$$
(\mathcal{J} \kappa)(x)-\left(C_{N, h} \mathcal{J} C_{N, h} \kappa\right)(x)=\mathrm{O}(\log N) \quad(N \rightarrow \infty) .
$$

Proof. We have

$$
\begin{aligned}
& \left|(\mathcal{J} \kappa)(x)-\left(C_{N, h} \mathcal{J} C_{N, h} \kappa\right)(x)\right| \\
& \leq\left|(\mathcal{J} \kappa)(x)-\left(C_{N, h} \mathcal{J} \kappa\right)(x)\right|+\left|\left(C_{N, h} \mathcal{J} \kappa\right)(x)-\left(C_{N, h} \mathcal{J} C_{h} \kappa\right)(x)\right| \\
& \quad+\left|\left(C_{N, h} \mathcal{J} C_{h} \kappa\right)(x)-\left(C_{N, h} \mathcal{J} C_{N, h} \kappa\right)(x)\right| .
\end{aligned}
$$

First, the second and third terms on the right-hand side of (5.26) are bounded. This is because we can apply estimates similar to (5.22) and (5.23) in the proof of Theorem 2.3. for the second and third terms, respectively. Next, it follows from $\sup _{-\infty<x<\infty}|(\mathcal{J} \kappa)(x)|<\infty$ and the arguments similar to those used in (5.23) that the first term of the right-hand side of (5.26) is bounded by $c \log N$ for some $c$.

Let

$$
\tilde{r}_{N}=\left(h \sum_{k=-N}^{N} f(k h)\right) \kappa \text { and } \quad \tilde{g}_{N}=f-\tilde{r}_{N} .
$$

Then the quadrature formula in Theorem 2.5 can be written as

$$
\left(\mathcal{J} \tilde{r}_{N}\right)(x)+\left(C_{N, h} \mathcal{J} C_{N, h} \tilde{g}_{N}\right)(x),
$$

and the difference between it and the quadrature formula in Theorem 2.4 can be written as

$$
\left(\mathcal{J}\left(\tilde{r}_{N}-r\right)\right)(x)+\left(C_{N, h} \mathcal{J} C_{N, h}\left(\tilde{g}_{N}-g\right)\right)(x) .
$$

Hence, to prove (2.44), it suffices to show that

$$
\begin{aligned}
\left(\mathcal{J}\left(\tilde{r}_{N}-r\right)\right)(x) & +\left(C_{N, h} \mathcal{J} C_{N, h}\left(\tilde{g}_{N}-g\right)\right)(x) \\
& =\text { o }\left(\exp \left[\frac{-\pi\left(d_{g}-\varepsilon\right) \gamma_{g} N}{\log \left(\pi\left(d_{g}-\varepsilon\right) \gamma_{g} N / \beta_{g}\right)}\right]\right) .
\end{aligned}
$$


However, this can easily be derived from Proposition 5.3 and Lemma 5.7 as follows:

$$
\begin{aligned}
& \left|\left(\mathcal{J}\left(\tilde{r}_{N}-r\right)\right)(x)+\left(C_{N, h} \mathcal{J} C_{N, h}\left(\tilde{g}_{N}-g\right)\right)(x)\right| \\
& \quad=\left|(\mathcal{J} \kappa)(x)-\left(C_{N, h} \mathcal{J} C_{N, h} \kappa\right)(x)\right|\left|\int_{-\infty}^{\infty} f(t) \mathrm{d} t-h \sum_{k=-N}^{N} f(k h)\right| \\
& \quad \leq c^{\prime} \log N \exp \left[\frac{-2 \pi d_{f} \gamma_{g} N}{\log \left(\pi\left(d_{g}-\varepsilon\right) \gamma_{g} N / \beta_{g}\right)}\right] \\
& \quad=\mathrm{o}\left(\exp \left[\frac{-\pi\left(d_{g}-\varepsilon\right) \gamma_{g} N}{\log \left(\pi\left(d_{g}-\varepsilon\right) \gamma_{g} N / \beta_{g}\right)}\right]\right) .
\end{aligned}
$$

5.3. Explanation of the argument in subsection 2.4. Some details of the argument in subsection 2.4 are provided here. First note that (2.36) and (2.37) imply that

$$
\gamma_{g} d_{g} \leq \gamma_{f} d_{f}
$$

where $\gamma_{f} d_{f} \leq \pi / 2$ by Proposition 2.2, We divide the argument into two cases according to the value of $\gamma_{f} d_{f}$.

Case $1\left(\gamma_{f} d_{f}<\pi / 2\right)$. We take $B$ and $C$ as (2.47) and (2.48), respectively. Then, we have

$$
\begin{aligned}
\gamma_{g} & =\min \left\{\gamma_{f}, C\right\}=\gamma_{f}, \\
d_{g} & =\min \left\{d_{f}, \frac{1}{C} \arcsin \left[\frac{\pi}{2 B}\right]-\varepsilon_{d}\right\} \\
& =\min \left\{d_{f}, \frac{1}{\gamma_{f}} \arcsin \left[\frac{\sin \left(\gamma_{f} d_{f}\right)}{1-\left(2 \varepsilon_{B} / \pi\right) \sin \left(\gamma_{f} d_{f}\right)}\right]-\varepsilon_{d}\right\}=d_{f},
\end{aligned}
$$

because $\varepsilon_{B}>0$ and $\varepsilon_{d}$ is sufficiently small. Hence we have that $\gamma_{g} d_{g}$ attains the upper bound $\gamma_{f} d_{f}$ in (5.29). Note that this upper bound is attained only when $\gamma_{g}=\gamma_{f}$ and $d_{g}=d_{f}$, and this, in turn, implies that

$$
0<B<\frac{\pi}{2}
$$

or

$$
\frac{\pi}{2} \leq B<\frac{\pi}{2 \sin \left(\gamma_{f} d_{f}\right)}
$$

where the latter follows from

$$
\pi / 2 \leq B, \gamma_{f} \leq C, d_{f}<(1 / C) \arcsin (\pi /(2 B)) .
$$

Thus $\beta_{g}=\min \left\{\beta_{f}, B-\varepsilon_{\beta}\right\}$ is nearly optimal. 
Case $2\left(\gamma_{f} d_{f}=\pi / 2\right)$. Since

$$
\gamma_{g} d_{g} \leq \gamma_{\kappa} d_{\kappa}<\frac{\pi}{2}
$$

the upper bound $\gamma_{f} d_{f}$ in (5.29) cannot be attained in the case of $\gamma_{f} d_{f}=\pi / 2$. However, $\gamma_{g} d_{g}$ can be made arbitrarily close to $\pi / 2$ with the choice of $B=\pi / 2$ and $C=\gamma_{f}$ given in (2.52) and (2.53), for which we have

$$
\begin{aligned}
\gamma_{g} d_{g} & =\min \left\{\gamma_{f}, C\right\} \min \left\{d_{f}, \frac{\pi}{2 C}-\varepsilon_{d}\right\} \\
& =\gamma_{f} \min \left\{d_{f}, \frac{\pi}{2 \gamma_{f}}-\varepsilon_{d}\right\} \\
& =\frac{\pi}{2}-\varepsilon_{d} \gamma_{f} .
\end{aligned}
$$

To maximize $\beta_{g}=\min \left\{\beta_{f}, B-\varepsilon_{\beta}\right\}$ we are to choose $B$ as large as possible, while keeping $\gamma_{g} d_{g}$ invariant. Our choice of $B=\pi / 2$ is also optimal in this respect, since, for $B>\pi / 2$, we have

$$
\gamma_{f} d_{f}-\gamma_{g} d_{g}>\frac{\pi}{2}-\arcsin \left(\frac{\pi}{2 B}\right)>0 .
$$

\section{Concluding Remarks}

Formulas for numerical indefinite integration by sinc approximation with double exponential transformations are also considered in [5. These are based on Haber's formula (A) in 2], whereas our formula is based on Stenger's [6], which is similar to $(\mathrm{B})$ in [2].

The error bound of our formula depends on individual integrands. Theoretically it is more desirable to have a formula that is valid for functions in a certain function space and has a uniform error bound that depends only on the function space. A possible deeper theoretical problem is concerned with the optimality of the formula in the function space. Here, the optimality means the superiority of the formula in its accuracy over any other formula applicable to the elements of the function space. For example, such a problem has been addressed for a trapezoidal formula [8] and a sinc interpolation [9.

\section{REFERENCES}

[1] R. P. Boas, Entire functions, Academic Press, New York, 1954. MR0068627 (16:914f)

[2] S. Haber, Two formulas for numerical indefinite integration, Math. Comp. 60 (1993), 279296. MR 1149292 (93d:65026)

[3] R. B. Kearfott, A sinc approximation for the indefinite integral, Math. Comp. 41 (1983), 559-572. MR0717703 (85g:65029)

[4] M. Mori and M. Sugihara, The double-exponential transformation in numerical analysis, in: Numerical Analysis in the 20th Century, Vol. V: Quadrature and Orthogonal Polynomials, J. Comput. Appl. Math. 127 (2001), 287-296. MR.1808579 (2001k:65041)

[5] M. Muhammad and M. Mori, Double exponential formulas for numerical indefinite integration, J. Comput. Appl. Math. 161 (2003), 431-448. MR.2017024

[6] F. Stenger, Numerical methods based on sinc and analytic functions, Springer-Verlag, New York, 1993. MR 1226236|(94k:65003) 
[7] F. Stenger, Summary of sinc numerical methods, in: Numerical Analysis in the 20th Century, Vol. I: Approximation theory, J. Comput. Appl. Math. 121 (2000), 379-420. MR1780056 (2001d:65018)

[8] M. Sugihara, Optimality of the double exponential formula-functional analysis approach, Numer. Math. 75 (1997), 379-395. MR1427714 (97i:41041)

[9] M. Sugihara, Near optimality of the sinc approximation, Math. Comp. 72 (2003), 767-786. MR 1954967 (2004a:41026)

[10] H. Takahasi and M. Mori, Double exponential formulas for numerical integration, Publ. Res. Inst. Math. Sci. Kyoto Univ. 9 (1974), 721-741. MF0347061 (49:11781)

Department of Mathematical Informatics, Graduate School of Information Science and Technology, University of TOKYo, Tokyo 113-8656, Japan

E-mail address: kenitiro@misojiro.t.u-tokyo.ac.jp

Department of Computational Science and Engineering, School of Engineering, NAgOYA University, NAgOYA 464-8603, JAPAN

E-mail address: sugihara@na.cse.nagoya-u.ac.jp

Department of Mathematical Informatics, Graduate School of Information Science and Technology, University of TOKYo, Tokyo 113-8656, Japan

E-mail address: murota@mist.i.u-tokyo.ac.jp 